\title{
A New Parallel Matrix Multiplication Algorithm on Tree-Hypercube Network using IMAN1 Supercomputer
}

\author{
Orieb AbuAlghanam, \\ Mohammad Qatawneh \\ Computer Science Department \\ University of Jordan \\ Amman-Jordan
}

\author{
Hussein A. al Ofeishat \\ Computer Science Department \\ Al-Balqa applied university Jordan \\ Amman-Jordan
}

\author{
Omar Adwan, Ammar Huneiti \\ Computer Information Systems \\ University of Jordan \\ Amman-Jordan
}

\begin{abstract}
The tree-hypercube (TH) interconnection network is relatively a new interconnection network, which is constructed from tree and hypercube topologies. TH is developed to support parallel algorithms for solving computation and communication intensive problems. In this paper, we propose a new parallel multiplication algorithm on TH network to present broadcast communication operation for TH using store-and-forward technique, namely, one-to-all broadcast operation which allows a message to be transmitted through the shortest path from the source node to all other nodes. The proposed algorithm is implemented and evaluated in terms of running time, efficiency and speedup with different data size using IMAN1. The experimental results show that the runtime, efficiency and the speedup of the proposed algorithm decrease as a number of processors increases for all cases of matrices size of $1000 \times 1000$, $2000 \times 2000$, and $4000 \times 4000$.
\end{abstract}

Keywords-MPI; supercomputer; tree-hypercube; matrix multiplication

\section{INTRODUCTION}

Parallel matrix multiplication is considered as a backbone for several scientific applications. Many studies developed matrix multiplication from different perspective in [3], [6], [7], [10], [14] the authors studied the limitation bandwidth for memory-processor through communication and how to reduce the gap between memory and processor speed, they present a communication efficient mapping of a large-scale matrix multiplication algorithm. In [1] parallel matrix multiplication algorithm on hypercube multiprocessors, while in [2], [5], [6] the authors applied a matrix multiplication with a hypercube algorithm on multi-core processor cluster. In [3] the author proposed a Distribution-Independent Matrix Multiplication Algorithm called DIMMA which is new, fast, and scalable matrix multiplication algorithm, for block cyclic data distribution on distributed-memory concurrent computers. In this paper we apply matrix multiplication on tree-hypercube which was used before in adaptive fault tolerate in routing algorithm [8], [11], [12], [17].

Many proposals for matrix multiplication algorithms were done on different networks type whether it was homogeneous or heterogeneous in order to reduce the time drastically to improve the system performance. Matrix multiplication needs high computation time especially when the size of the matrix becomes huge, therefore some problems need years to be solved using a personal computer. The interconnection networks are the core of a parallel processing system which the system's processors are linked. Due to the big role played by the networks topology to improve the parallel system's performance, several interconnection network topologies have been proposed for that purpose; such as the tree, hypercube, mesh, ring, and Hex-Cell (HC) [12], [13], [16].

Among the wide variety of interconnection networks structures proposed for parallel computing systems is TreeHypercube network which received much attention due to the attractive properties inherited in their topology [4], [8], [9], [15].

This paper aimed to design and analyze efficient matrix multiplication algorithm on tree-hypercube network. Experimentation of the proposed algorithm was conducted using IMAN1 supercomputer which is Jordan's first supercomputer. The IMAN1 is available for use by academia and industry in Jordan and the region.

The rest of the paper is organized as follows. Section 2 summarizes the definition of Tree-Hypercube network. In Section 3 the proposed algorithm is explained, Section 4 is an overview of sequential and parallel matrix multiplication is discussed. In Section 5, the evaluation results are illustrated. Finally, section 6 is the conclusion for the paper.

\section{DEFINITION OF TREe-HyPERCUBE NETWORK}

Tree-hypercube can be defined as follows: each node is labelled with the level number (L) and the node number (X) with the form $(\mathrm{L}, \mathrm{X})$. It is mainly a tree which represents a hypercube in each level because each level $\mathrm{i}$ in tree has s(i) nodes that represents the number of processing element as a hypercube [4], [9]. For very level L between 0 and d, each node $(\mathrm{L}, \mathrm{X})$ in level $\mathrm{L}$, where $\mathrm{X}=\mathrm{X}(\mathrm{d} \operatorname{logs})-1 \ldots \ldots \mathrm{X} 0$ is adjacent to the following $s$ children nodes at level $\mathrm{L}+1(\mathrm{~L}+1$, X.a) for a $=0, \ldots . ., \mathrm{s}-1$ as shown in Fig. 1(a) and (b) [8]. 


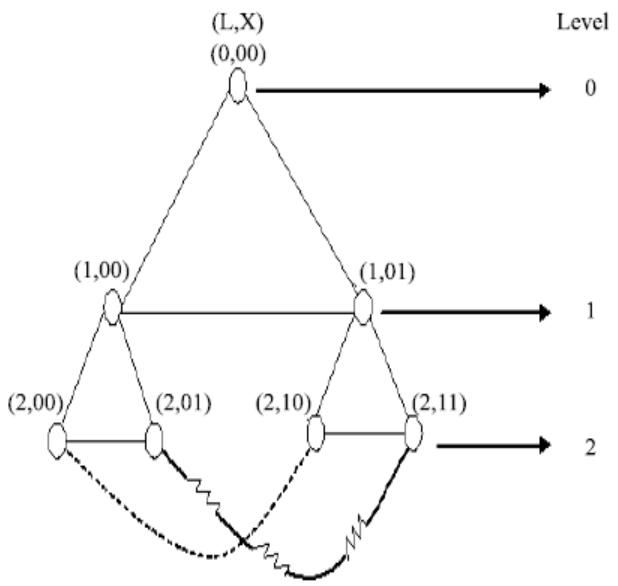

(a)

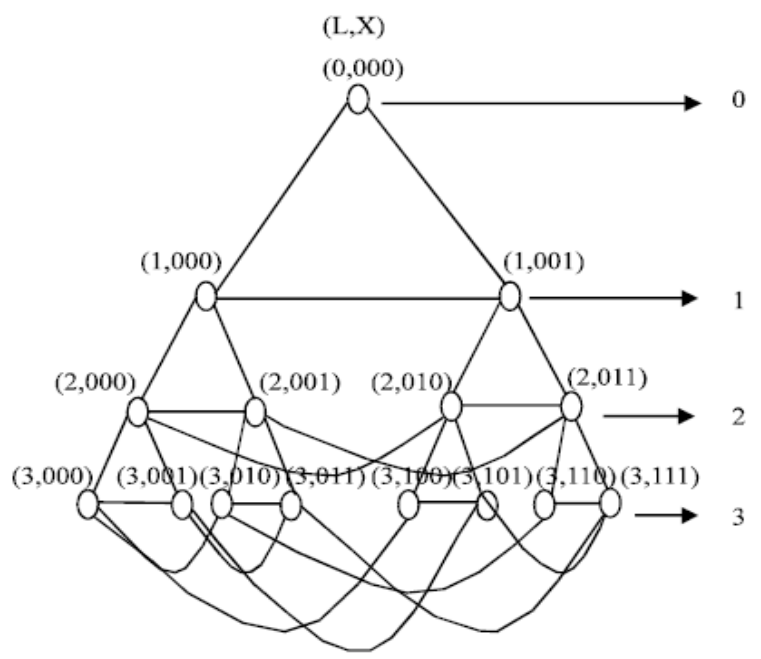

(b)

Fig. 1. Tree-hypercube (a) $\mathrm{TH}(2,2)$ and (b) $\mathrm{TH}(2,3)$.

\section{PROPOSED ALGORITHM}

Matrix multiplication is a simple and widely used algorithm in many scientific applications. Matrix multiplication is well structured in the sense that elements of the matrices can be evenly distributed to the nodes and communication among nodes which have regular pattern. Therefore, exploiting data parallelism based on message passing is suitable for solving the matrix multiplication problem. Fig. 2 shows a sequential algorithm of an $n \times n$ matrix multiplication consisting of three nested loops. As can be seen, the complicity of the algorithm is $n 3$.

$$
\begin{aligned}
& \text { Matrix-Multiplication(A,B) } \\
& \mathrm{n}=\mathrm{A} \text {.rows } \\
& \text { Let } \mathrm{C} \text { be a new } \mathrm{n} \times \mathrm{n} \text { matrix } \\
& \text { For } \mathrm{i}=1 \text { to } \mathrm{n} \\
& \text { For } \mathrm{j}=1 \text { to } \mathrm{n} \\
& \mathrm{Cij}=0 \\
& \text { For } \mathrm{k}=1 \text { to } \mathrm{n} \\
& \mathrm{Cij}=\mathrm{Cij}+\text { aik bkj }
\end{aligned}
$$

In this section, we propose a new Parallel Matrix Multiplication Algorithm on Tree-Hypercube Network Using IMAN1 Supercomputer. The data distribution for matrix multiplication can be divided either striped partitioning or checkerboard partitioning. We use a block striped partitioning distribution to study the performance of message passing execution model. The column wise block striping of an ( $\mathrm{x} \times \mathrm{n}$ ) matrix on $\mathrm{p}$ processors $(\mathrm{P} 0, \mathrm{P} 1, \ldots, \mathrm{Pp}-1)$ processor $\mathrm{Pi}$ contains columns: (n/p) (i), ((n/p) i) $+1, \ldots,((n / p)(i+1))-1$ [5].The proposed parallel matrix multiplication algorithm on Tree-Hypercube network consists of three stages as shown in Fig. 3. The first stage shows the distribution of data among nodes (processors), second stage describe the multiplication process at each processor, and finally the third stage represent the collecting the data.

\section{Input: Matrix A and B \\ Output: Matrix $\mathrm{C}$ on Tree-Hypercube using parallel Matrix Multiplication}

\section{Stage 1: Broadcast matrix A and B}

1. CN (Coordinator Node) generates a set of blocks of Matrix A.

2. CN generates a set of blocks according to the Matrix B.

3. Wait for acknowledgment message from the coordinator who received the data.

4. Send a message for the $\mathrm{CN}$ informing the process completion.

5. $\mathrm{CN}$ stops the process of distribution and announces the beginning of the next Stage

\section{Stage 2: Do the multiplication in parallel}

6. Multiply the stripes of matrix A with stripes of Matrix B (for each block) of data.

7. Using sequential matrix Multiplication. Where all processors perform $\mathrm{Cij}=\sum_{k=0}^{k-1} A(\boldsymbol{i} k) B(k j)$

\section{Stage 3: Collecting the data}

A- Global Data Combining

8. In each level in the Tree-Hypercube interconnection, send in parallel the multiplication matrix to the TH root nodes.

B- Combining Data in the Tree-Hypercube root nodes

9. $\mathrm{CN}$ combines the collected matrices correctly from roots nodes in matrix $\mathrm{C}$.

10. Exit

Fig. 3. Pseudocode Tree-Hypercube Algorithm for Matrix Multiplication.

Fig. 2. A Sequential Multiplication Algorithm for two matrices [18]. 


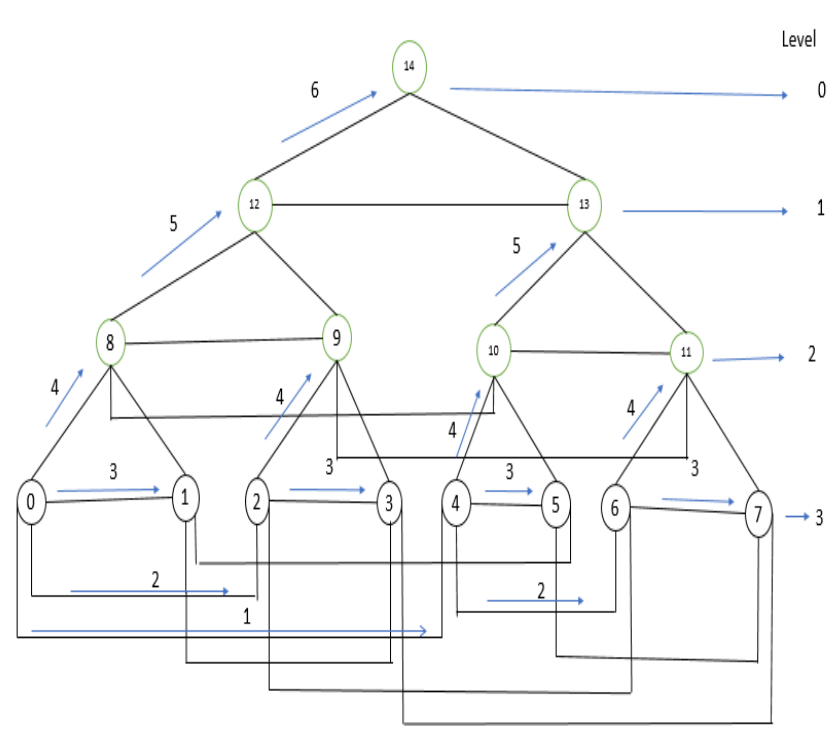

Fig. 4. Parallel matrix multiplication tree-hypercube view.

Fig. 4 shows one-to-all broadcast on a Tree-Hypercube $(2,3)$. Broadcast communication operations are used in many parallel algorithms; such as vector-matrix multiplication, vector-vector multiplication, matrix multiplication, Gaussian elimination, shortest paths, LU (lower-upper) factorization, maximal vectors and prefix sums. Our one-to-all broadcast algorithms on TH network are based on the E-Cube routing algorithm, where this routing algorithm can be found in [1]. Each node can send a message while receiving another message on the same or different link; that is bidirectional links are used. Also, it can send or receive a message on only one of its links at a time. Moreover, a store-and-forward routing technique is used, where each intermediate node must fully receive and store the entire message before sending it to the next node. Fig. 4 presents one-to-all broadcast algorithm which can be defined as broadcasting a message from a source node to all other nodes in the network. Respectively, in matrix multiplication which is applied in tree hypercube, the coordinator creates the partitions depending on the number of processors and the matrix size, once the data is received by any processor it takes the part that it is responsible for and then resends it again to the nodes that has a direct link with it. In TH sending data acts as hypercube in each level, after the data distribution is done. Each processor does it is own computations and sends back the result to the parent from which it got the data until all data is accumulated and sent back to the coordinator.

\section{A. Partition Procedure Analysis}

In the first partitioning of the original array will take $n / p$, assuming the partitioning is always balanced i.e. the number of columns or the numbers of rows are equal to the number of processors.

In our tree-hypercube network only one processor has data, which is called the coordinator. In the first step part of data will be sent from the coordinator to the directly connected processor, secondly, both processors will start sending other parts of data to the processors that are connected directly to them. TH $(2,2)$, each processor will receive a row form matrix $\mathrm{A}$ and a column from matrix B, assuming the matrix size is 7x7. Canon algorithm is a distributed algorithm for matrix multiplication for two-dimensional meshes. It is especially suitable for computers laid out in an $\mathrm{N} \times \mathrm{N}$ mesh and it works well in homogeneous $2 \mathrm{D}$ grids, so the main advantage of the algorithm is that its storage requirements remain constant and are independent on the number of processors. As shown in Fig. 5, the matrix multiplication using Canon algorithm with 49 processor, it aligns the blocks of $\mathrm{A}$ and $\mathrm{B}$ in such a way that each process multiplies its local sub-matrices. This is done by shifting all sub-matrices Ai,j to the left (with wraparound) by i steps and all sub-matrices $\mathrm{Bi}, \mathrm{j}$ up (with wraparound) by $\mathrm{j}$ steps [5].

\section{B. Parallel Analysis}

Parallel matrix multiplication is analyzed according to the communication time, computation time and complexity. Communication steps involve the distribution of data splitter between the processors, and gathering results. First of all, the number of processors will affect both the data scatter and the communication time, to calculate the initialization time for the tree-hypercube $\mathrm{TH}$. The initialization time will equal the number of steps to distribute data between the processor units. We need 4 steps to scatter data among 7 processors and 6 steps for 15 processors, so we need (2L) for initialization time and the same time to gather the results from all processors. So, the total communication time equals (2L).

Complexity is the time required to perform matrix multiplication in each processor for data size $(\mathrm{n} / \mathrm{p})$ which is required for all processors, this means each processor needs $(\mathrm{n} / \mathrm{p}) \times\left(n^{3}\right)$ time, which makes the total complexity $\left(\left(n^{4}\right) / \mathrm{p}\right)$.

Execution time depends on the number of processor and the size of data as discussed previously the complexity is the time needed for multiplication and the communication time which is $(2 \mathrm{~L})+\left(\left(n^{2}\right) /\left(p^{2}\right)\right)$.

\begin{tabular}{|c|c|c|c|c|c|c|c|c|c|c|c|c|c|}
\hline $\mathrm{A} 00$ & A06 & A05 & A04 & $\mathrm{A} 03$ & A02 & \begin{tabular}{|l|} 
A01 \\
\end{tabular} & BO0 & \begin{tabular}{|l|} 
B61 \\
\end{tabular} & B52 & B43 & B34 & \begin{tabular}{|l|} 
B25 \\
\end{tabular} & B16 \\
\hline A16 & A15 & A14 & A13 & A12 & A11 & A10 & $\mathrm{B} 60$ & B51 & B42 & B33 & B24 & B15 & B06 \\
\hline A25 & A24 & A23 & A22 & A21 & A20 & A26 & B50 & B41 & B32 & B23 & B14 & B05 & B66 \\
\hline A34 & A33 & A32 & A31 & A30 & A36 & A35 & B40 & B31 & B22 & B13 & B04 & B65 & B56 \\
\hline A43 & A42 & A41 & A40 & A46 & A45 & A44 & B30 & B21 & B12 & B03 & B64 & B55 & B46 \\
\hline A52 & A51 & A50 & A56 & A55 & A54 & A53 & B20 & B11 & B02 & B63 & B54 & B45 & B36 \\
\hline A61 & A60 & A66 & A65 & A64 & A63 & A62 & B10 & B01 & B62 & \begin{tabular}{|l|l|} 
\\
\end{tabular} & \begin{tabular}{|l|l|} 
\\
\end{tabular} & B35 & B26 \\
\hline
\end{tabular}

Fig. 5. Initial data distribution of matrix A $7 \times 7$, B $7 \times 7$ and $C 7 \times 7$ on 49 processors $(2,2)$. 


\section{EVOLUTION RESULT}

Results are evaluated in terms of speedup, running time and the parallel efficiency of matrix multiplication. The proposed parallel matrix multiplication on the Tree-Hypercube network is implemented by the library Message Passing Interface MPI, in which MPI processes are assigned to the cores. It will be parallel computation if the MPI process is assigned to a core; but if more than one MPI process is assigned to the same core, then it will be concurrent computation. These results were produced in IMAN1 Zaina cluster. Also, open access MPI library was used in our implementation. The experiment was applied on treehypercube topology with different size of matrices and different number of processors in reference to the number of nodes in a tree-hypercube, for example in the below table the number of processors represent the number of nodes inside tree-hypercube definition. After running this experiment multiple times, the average was taken as result. The hardware and software specifications are illustrated in Table I.

TABLE I. HARDWARE AND SOFTWARE SPECIFICATION

\begin{tabular}{|l|l|}
\hline Hardware specification & $\begin{array}{l}\text { Dual Quad Core Intel Xeon CPU with SMP } \\
\text { 16 GB RAM }\end{array}$ \\
\hline Software specification & $\begin{array}{l}\text { Scientific Linux 6.4 with open MPI1.5.4 } \\
\text { and C++ compiler }\end{array}$ \\
\hline Matrix size & $1000 \times 1000,2000 \times 2000,4000 \times 4000$ \\
\hline Number of processors & $1,3,7,15,31$ \\
\hline
\end{tabular}

\section{A. Run Time Evaluation}

Fig. 6 depicts run time for sequential matrix multiplication in different size $1000 \times 1000,2000 \times 2000$ and $4000 \times 4000$ we can see as the matrix size increase the runtime increases proportionally.

Fig. 7 shows the run time for Parallel matrix multiplication in different sizes $1000 \times 1000,2000 \times 2000$ and $4000 \times 4000$ as illustrated in Fig. 7 when the number of processes increases the time needed for multiplication decreases. On the other hand, as shown below the run time at a certain number of processes will stop decreasing because the problem becomes smaller than the number of processors and the communication time will increase because this will cause an overhead, the general behaviour can be summarized with the following points:

Scenario 1: When the processors number increases the run time decreases due to parallelism and distribution of tasks on more than one processor that are working together at the same time. (This case is applied when we move from 3 processors to 31 processors.)

Scenario 2: When the processors number increases the run time increases due to communication overhead which is caused from increasing the number of processors more than a specific data size which in its turn decreases the benefits of the parallelism. (This case is applied when we move from 15 processors to 31 processors in $1000 \times 1000$ matrices and also may occur in number of processors larger than 31 in $2000 \times 2000$ and $4000 \times 4000$ matrices.)

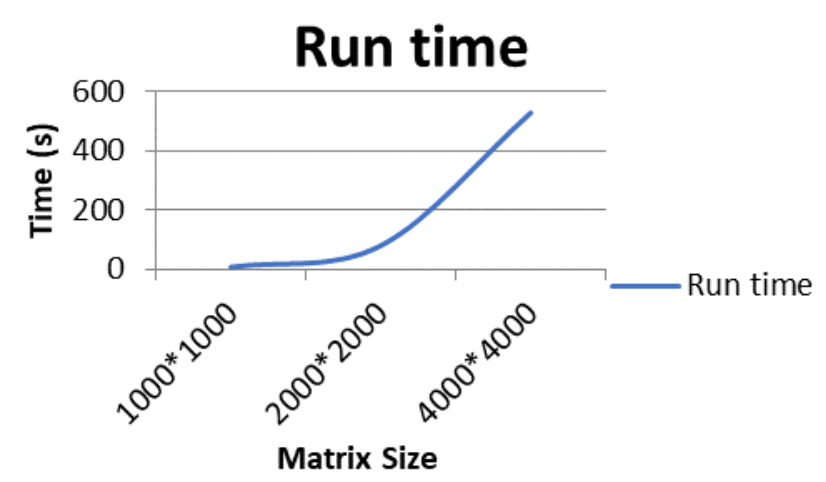

Fig. 6. Runtime for sequential matrix multiplication with different matrix size.

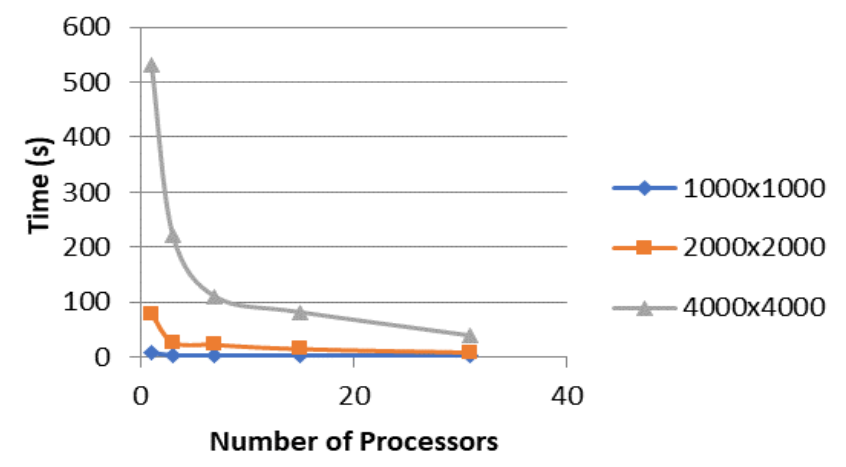

Fig. 7. Runtime of matrix multiplication according to the number of processors and with different matrix size.

\section{B. Speed up Evaluation}

The speedup is the ratio of serial to parallel execution time as depicted in Fig. 8. Using (1) the number of processors values tested were $3,7,15$ and 31 . The speedup increases when the number of processors increases but this is not always applicable because we have limitations for parallelism that appears in matrices with size $1000 \times 1000$.

Speedup $=$ sequential processing time/ parallel processing time

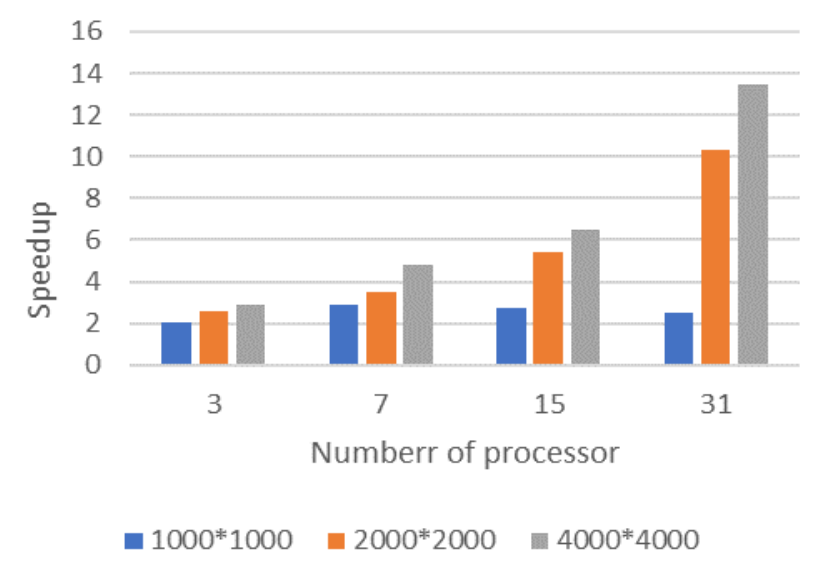

Fig. 8. The Speedup of the three different sizes of matrices on different number of processors. 


\section{Parallel Efficiency Evaluation}

Efficiency is the ratio of speedup to the number of processors. In an ideal parallel system $\mathrm{S}=\mathrm{P}$ and $\mathrm{E}=1$ but in practice $\mathrm{S}<\mathrm{P}$ and $\mathrm{E}$ is between 0 and 1 . Equation 2 is used to calculate the efficiency. Fig. 9 displays the parallel efficiency for matrix multiplication according to different number of processors and different matrix size. In small numbers of processors between (3 and 7) efficiency was $97 \%$ for all matrix multiplication of matrix size $4000 \times 4000$. On the other hand, when the number of processors increased between (15 and 31) efficiency started to decrease i.e. reached $8 \%$ in $1000 \times 1000$ with 31 processors because the communication time will increase between the processes. Total communication time equals $(2 \mathrm{~L})$, so the communication time will increase when the number of processors increases.

The reason why the efficiency goes down, is that the communication time equals $2 \mathrm{~L}$ where $\mathrm{L}$ is number of levels for tree hypercube, as long as the communications processes are increasing the communication time will increase for example when the number of processers is 3 the communication time will be 2 on the other hand when we have 31 processors the communication time will be 8 seconds for the same data size. Also, as seen in equation 2 the number of processes is in inverse relationship with efficiency.

$$
\text { Efficiency }=\text { speedup } / \text { number of processors }
$$

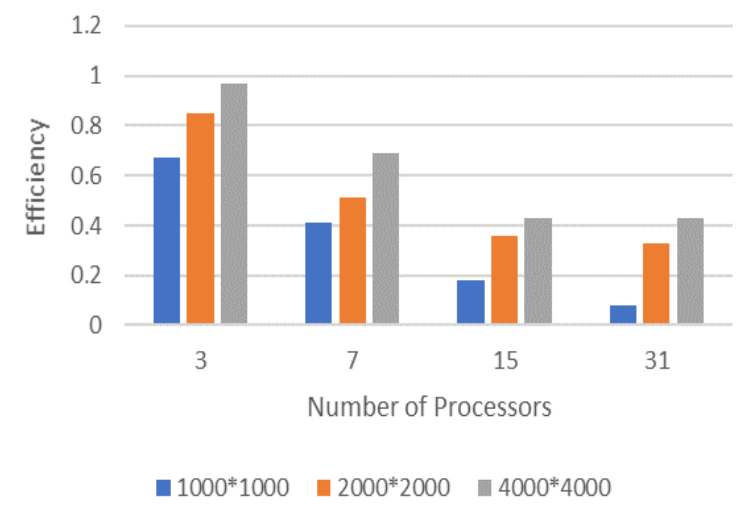

Fig. 9. Efficiency of the three different sizes of matrices on different number of processors.

\section{CONClUSIONS AND FUTURE WORKS}

In this paper, we present Matrix multiplication using treehypercube in terms of running time, speedup and efficiency. The algorithm was implemented using MPI library, and the results were produced by IMAN1 super computer. The evaluation was based on matrix size and number of processors. Results showed that runtime was in general decreasing when the processer's number is increased except for matrix size $1000 \times 1000$ when the number of processors exceeded 15 the runtime increased because of the communication overhead. The speedup in all different matrices size increased when the number of processors increased except for the cases in which the communication overhead increases. We achieved up to $97 \%$ efficiency in large matrices such as $4000 \times 4000$ sized matrix.
In our future work we are aiming to proceed with a comparative study for matrix multiplication using different interconnection networks and it will be applied also on different algorithms such as sorting, then see the performance for each and compare the results with different studies.

\section{REFERENCES}

[1] P. Lee, "Parallel Matrix Multiplication Algorithms on Hypercube Multiprocessors", International Journal of High Speed Computing, (1995)

[2] J. Zavala-Díaz, J. Pérez-Ortega, E. Salazar-Reséndiz, and L. Guadarrama-Rogel matrix,"multiplication with a hypercube algorithm on multi-core processor cluster", DYNA,Vol (82), no(191). 2015

[3] J. Choi,"New Parallel Matrix Multiplication Algorithm on DistributedMemory" ,Concurrent Computers. High Performance Computing on the Information Superhighway, 1997. HPC Asia '97.Pages: 224 229, DOI: 10.1109/HPC.1997.592151

[4] M. Al-Omari, and H. Abu-Salem, "Tree-hypercubes: A multiprocessor interconnection topology", Abhath Al-Yarmouk, 6: 9-24. (1997).

[5] A. Grama, G. Karypis, and V. Kumar, "Introduction to Parallel Computing," Second Edition. Addison Wesley (2003).

[6] S. Panigrahiand, S. Chakraborty, "Statistical Definition of an Algorithm in PRAM Model \& Analysis of $2 \times 2$ Matrix Multiplication in $2 n$ Processors Using Different Networks", IEEE International Advance Computing Conference $\quad 717$ 724, DOI: 10.1109/IAdCC.2014.6779412. (2014)

[7] A. Haron, J. Yu, R. Nane, M. Taouil, S. Hamdioui, and K. Bertels, "Parallel Matrix Multiplication on Memristor-Based Computation-inMemory Architecture". International Conference on High Performance $\begin{array}{llll}\text { Computing } \& \text { Simulation } & \text { (HPCS)Pages: 759- }\end{array}$ 766, DOI: 10.1109/HPCSim.2016.7568411

[8] M. Qatawneh, "Adaptive Fault Tolerant Routing Algorithm for Tree Hypercube Multicomputer".vol. 2, no. 2, pp. 124-126. (2006).

[9] M. Qatawneh. "Embedding Linear Array Network into the treehypercube Network", European Journal of Scientific Research, 10(2). 2005, pp. $72-76$.

[10] Md. Nazrul Islam, Md. Shohidul Islam, M.A. Kashem, M.R. Islam, M.S. Islam "An Empirical Distributed Matrix Multiplication Algorithm to Reduce Time Complexity", Proceedings of the International MultiConference of Engineers and Computer Scientists Vol IIIMECS 2009, March 18 - 20, 2009, Hong Kong).

[11] M. Qatawneh. "Multilayer Hex-Cells: A New Class of Hex-Cell Interconnection Networks for Massively Parallel Systems.", International journal of Communications, Network and System Sciences, 4(11). 2011.

[12] M. Qatawneh. "Embedding Binary Tree and Bus into Hex-Cell Interconnection Network", Journal of American Science, 7(12). 2011.

[13] M. Qatawneh. "New Efficient Algorithm for Mapping Linear Array into Hex-Cell Network", International Journal of Advanced Science and Technology, 90, 2016.

[14] M. Saadeh, H. Saadeh, M. Qatawneh, "Performance Evaluation of Parallel Sorting Algorithms on IMAN1 Supercomputer", International Journal of Advanced Science and Technology Vol.95 (2016), pp.57-72.

[15] M. Qatawneh, A. Alamoush, J. Alqatawna. "Section Based Hex-Cell Routing Algorithm (SBHCR)", International Journal of Computer Networks \& Communications (IJCNC), 7(1). 2015.

[16] Qatawneh Mohammad, Hebatallah Khattab. "New Routing Algorithm for Hex-Cell Network", International Journal of Future Generation Communication and Networking, 8(2). 2015.

[17] M. Qatawneh, A. Sleit, W. Almobaideen. "Parallel implementation of polygon clipping using transputer", American journal of Applied Science 6 (2). 2009.

[18] A.Grama, A. Gupta, G.Karypis and V.Kumar. . "Introduction to Parallel Computing," second edition, Addison Wesley,January 16, 2003. ISBN: 0-201-64865-2. 